\title{
More on the infrared renormalization group limit cycle in QCD
}

\author{
E. Epelbaum* \\ Jefferson Laboratory, Theory Division, Newport News, VA 23606, USA \\ H.-W. Hamment \\ Helmholtz-Institut für Strahlen- und Kernphysik (Theorie), \\ Universität Bonn, Nußallee 14-16, D-53115 Bonn, Germany \\ Ulf-G. Meißner ${ }^{\sharp}$ \\ Helmholtz-Institut für Strahlen- und Kernphysik (Theorie), \\ Universität Bonn, Nußallee 14-16, D-53115 Bonn, Germany and \\ Institut für Kernphysik (Theorie), Forschungszentrum Jülich, D-52425 Jülich, Germany \\ A. Nogga \\ Institut für Kernphysik (Theorie), Forschungszentrum Jülich, D-52425 Jülich, Germany
}

(Dated: February 2, 2008)

\begin{abstract}
We present a detailed study of the recently conjectured infrared renormalization group limit cycle in QCD using chiral effective field theory. It was conjectured that small increases in the up and down quark masses can move QCD to the critical trajectory for an infrared limit cycle in the three-nucleon system. At the critical quark masses, the binding energies of the deuteron and its spin-singlet partner are tuned to zero and the triton has infinitely many excited states with an accumulation point at the three-nucleon threshold. We exemplify three parameter sets where this effect occurs at next-to-leading order in the chiral counting. For one of them, we study the structure of the three-nucleon system in detail using both chiral and contact effective field theories. Furthermore, we investigate the matching of the chiral and contact theories in the critical region and calculate the influence of the limit cycle on three-nucleon scattering observables.
\end{abstract}

PACS numbers: 12.38.Aw, 21.45.+v, 11.10.Hi

Keywords: Renormalization group, limit cycle, quantum chromodynamics

*Electronic address: epelbaum@jlab.org

${ }^{\dagger}$ Electronic address: hammer@itkp.uni-bonn.de

${ }^{\ddagger}$ Electronic address: meissner@itkp.uni-bonn.de

$\S$ Electronic address: a.nogga@fz-juelich.de 


\section{INTRODUCTION}

The renormalization group $(\mathrm{RG})$ is an important tool in many areas of physics. Its applications range from critical phenomena in condensed matter physics to the nonperturbative formulation of quantum field theories for elementary particles [1]. The requirement of the invariance of low-energy observables under changes of the ultraviolet cutoff $\Lambda$ generates a RG flow on the multidimensional space of coupling constants $\mathbf{g}$ for operators in the Lagrangian:

$$
\Lambda \frac{d}{d \Lambda} \mathbf{g}=\boldsymbol{\beta}(\mathbf{g})
$$

Depending on the physical application, the RG flow can be considered in the infrared $(\Lambda \rightarrow 0)$ or in the ultraviolet $(\Lambda \rightarrow \infty)$ limits. The simplest solution to the $\mathrm{RG}$ equations is a fixed point $\mathbf{g}_{*}$ which satisfies $\boldsymbol{\beta}\left(\mathbf{g}_{*}\right)=0$. An important signature of an $\mathrm{RG}$ fixed point is scale invariance: symmetry with respect to the coordinate transformation $\mathbf{r} \rightarrow \lambda \mathbf{r}$ for any positive number $\lambda$. This symmetry implies that dimensionless variables scale as powers of the momentum scale. Scale-invariant behavior at long distances, as in critical phenomena, can be explained by RG flow to an infrared fixed point. Scale-invariant behavior at short distances, as in asymptotically-free field theories, can be explained by $\mathrm{RG}$ flow to an ultraviolet fixed point.

However, RG equations can also exhibit more complicated solutions. The possibility of RG flow to a limit cycle was proposed by Wilson in 1971 [2]. A limit cycle is a 1-parameter family of coupling constants $\mathbf{g}_{*}(\theta)$ that is closed under the RG flow and can be parametrized by an angle $0<\theta<2 \pi$. The $R G$ flow carries the system around a complete orbit of the limit cycle every time the ultraviolet cutoff $\Lambda$ increases by some factor $\lambda_{0}$. One of the signatures of an RG limit cycle is discrete scale invariance: symmetry with respect to the coordinate transformation $\mathbf{r} \rightarrow \lambda_{0}^{n} \mathbf{r}$ only for integer values of $n$.

Before the fundamental theory of the strong interactions was known, Wilson had suggested that limit cycles might be relevant to the high-energy behavior of the strong interactions of elementary particles [2]. However, Quantum Chromodynamics (QCD) and asymptotic freedom were discovered soon thereafter [3] and the high-energy behavior of the strong interactions was explained by an ultraviolet fixed point in QCD. The low-energy structure of the strong interactions, however, is much more complicated and not dominated by the fixed point.

The low-energy sector can be described by exploiting the approximate chiral symmetry of QCD using chiral effective field theory (EFT) methods [4, 5, 6]. Chiral EFT is a powerful tool for analyzing the properties of hadronic systems at low energies in a systematic and model-independent way. It is formulated in an expansion around the chiral limit of QCD which governs low-energy hadron structure and dynamics. In chiral EFT's the quark mass dependence of the operators in the effective Lagrangian is included explicitly. Over the past 15 years, considerable progress has been made in understanding the structure of the nuclear force in this framework [7, 8, 9, 10, 11].

The quark mass dependence of the chiral nucleon-nucleon $(N N)$ interaction was studied with the primary aim to understand the chiral limit of nuclear physics [12, 13, 14]. While this question has not been fully resolved, these studies found that the inverse scattering lengths in the ${ }^{3} \mathrm{~S}_{1}-{ }^{3} \mathrm{D}_{1}$ and ${ }^{1} \mathrm{~S}_{0}$ channels both vanish if one extrapolates away from the physical limit 
to slightly larger quark masses. ${ }^{1}$ Subsequently, it was pointed out that QCD is close to the critical trajectory for an infrared RG limit cycle in the 3-nucleon sector. This led to the conjecture, that QCD could be tuned to the critical trajectory by small changes in the up and down quark masses away from their physical values [15]. The proximity of the physical quark masses to these critical values explains the successful description of the low-energy three-nucleon problem in terms of zero-range forces between nucleons initiated long ago [16]. The effective-field-theory formulation of this program exhibits an ultraviolet RG limit cycle 17. The proximity of physical QCD to the critical trajectory implies that the ultraviolet limit cycle of Ref. [17] is not just an artifact of the EFT but hints towards a limit cycle in QCD.

The connection between the limit cycle and three-nucleon observables is established by the Efimov effect [18] which occurs in the three-body sector of nonrelativistic particles with a resonant short-range $\mathrm{S}$-wave two-body interaction. The strength of the interaction is governed by the S-wave scattering length $a$. If $a$ is large and positive, there is a shallow two-body bound state with binding momentum $\kappa=1 / a$, if $a$ is large and negative there is a shallow virtual state characterized by the momentum scale $1 /|a|$. Efimov showed that if $|a|$ is much larger than the range $r_{0}$ of the interaction, there are shallow three-body bound states whose number increases logarithmically with $|a| / r_{0}$. In the resonant limit $a \rightarrow \pm \infty$, there are infinitely many shallow three-body bound states with an accumulation point at the three-body scattering threshold. If the particles are identical bosons, the ratio of the binding energies of successive states rapidly approaches the universal constant $\lambda_{0}^{2} \approx 515$. Efimov also showed that low-energy three-body observables for different values of $a$ are related by a discrete scaling transformation in which $a \rightarrow \lambda_{0}^{n} a$, where $n$ is an integer, and lengths and energies are scaled by the appropriate powers of $\lambda_{0}^{n}$ 18, 19]. The mathematical connection between the Efimov effect and RG limit cycles was first pointed out in Ref. [20].

The Efimov effect can also occur for fermions with at least three distinct spin or isospin states and therefore applies to nucleons as well. The spin-singlet and spin-triplet $n p$ scattering lengths are $a_{1 \mathrm{~S}_{0}}=-23.8 \mathrm{fm}$ and $a_{3 \mathrm{~S}_{1}}=5.4 \mathrm{fm}$. They are both significantly larger than the effective range, which is $r_{0}=1.8 \mathrm{fm}$ in the spin-triplet channel. Efimov used this observation as the basis for a qualitative approach to the three-nucleon problem [21]. A convenient implementation of this program is given by the so-called pionless or contact EFT [9, 22]. Nucleons are described as point particles with zero-range interactions whose strengths are adjusted to reproduce the scattering lengths $a_{3 \mathrm{~S}_{1}}$ and $a_{1} \mathrm{~S}_{0}$. The effective range and higher order terms in the low-energy expansions of the phase shifts are treated as perturbations. This approach works well in nuclear few-body systems dominated by momenta small compared to $M_{\pi}$. In the triton channel, the Efimov effect makes it necessary to include a three-body force at leading order in the power counting [17]. The three-body force can be fixed by using one three-body datum as input. All other three-body observables can then be predicted. The structure of this EFT is much simpler than the chiral EFT and the computational effort is considerably smaller. Since the contact EFT is based on an expansion around the limit of infinite scattering length, it is particularly well suited to describe processes governed by the large scattering length. As a consequence, the chiral and contact EFT's can mutually complement each other. A first exploratory study of the infrared limit

\footnotetext{
${ }^{1}$ Due to the nuclear tensor force, the ${ }^{3} \mathrm{~S}_{1}$ and ${ }^{3} \mathrm{D}_{1}$ channels are coupled. This mixing is included in the chiral EFT calculations while it appears as a higher order effect in the contact EFT discussed below. For simplicity, we will only refer to the ${ }^{3} \mathrm{~S}_{1}$ and ${ }^{1} \mathrm{~S}_{0}$ partial waves in the following.
} 
cycle in QCD in the contact EFT was carried out in Ref. [15]. The quark mass dependence of the nucleon-nucleon scattering lengths from Ref. [14] was used as input in this calculation.

In this paper, we study the possibility of an infrared limit cycle in QCD in more depth. We use both chiral EFT and pionless EFT and combine the strengths of both approaches. We use the chiral EFT to calculate the bound state spectrum of the triton in the vicinity of the limit cycle and study how well it is approximated by the contact EFT. Furthermore, we calculate various three-body scattering observables in pionless EFT and illustrate how they are affected by the limit cycle.

\section{CHIRAL EFFECTIVE FIELD THEORY}

The quark mass dependence of the chiral $N N$ interaction was calculated to next-toleading order (NLO) in the chiral counting in Refs. 12, 13, 14]. At this order, the quark mass dependence is synonymous to the pion mass dependence because of the Gell-MannOakes-Renner relation:

$$
M_{\pi}^{2}=-\left(m_{u}+m_{d}\right)\langle 0|\bar{u} u| 0\rangle / F_{\pi}^{2},
$$

where $\langle 0|\bar{u} u| 0\rangle \approx(-225 \mathrm{MeV})^{3}$ is the quark condensate. In the following, we will therefore refer only to the pion mass dependence which is more convenient for nuclear applications and treat the pion mass as a parameter that can be varied by adjusting the values of the quark masses. In the work of Refs. [12, 13, 14], it was found that the scattering lengths in the ${ }^{3} \mathrm{~S}_{1}-{ }^{3} \mathrm{D}_{1}$ and ${ }^{1} \mathrm{~S}_{0}$ channels both vanish in the pion mass region around $200 \mathrm{MeV}$.

In this work, we study the structure of the nuclear three-body system in this region of pion masses around $200 \mathrm{MeV}$. We will use the chiral $N N$ potential constructed from EFT using the method of unitary transformations [14]. To next-to-leading order (NLO) in the chiral power counting, this potential can be written as:

$$
V_{\mathrm{NLO}}=V^{\mathrm{OPE}}+V^{\mathrm{TPE}}+V^{\mathrm{cont}},
$$

where $V^{\mathrm{OPE}}, V^{\mathrm{TPE}}$, and $V^{\text {cont }}$ refer to the one-pion exchange, two-pion exchange, and contact potentials, respectively. They are given by the expressions:

$$
\begin{aligned}
& V^{\mathrm{OPE}}=-\frac{1}{4} \frac{g_{A}^{2}}{F_{\pi}^{2}}\left(1+2 \Delta-\frac{4 M_{\pi}^{2}}{g_{A}} \bar{d}_{18}\right) \boldsymbol{\tau}_{1} \cdot \boldsymbol{\tau}_{2} \frac{\left(\sigma_{1} \cdot \mathbf{q}\right)\left(\sigma_{2} \cdot \mathbf{q}\right)}{\mathbf{q}^{2}+M_{\pi}^{2}} \\
& V^{\mathrm{TPE}}=-\frac{\boldsymbol{\tau}_{1} \cdot \boldsymbol{\tau}_{2}}{384 \pi^{2} F_{\pi}^{4}}\left\{L(q)\left[4 M_{\pi}^{2}\left(5 g_{A}^{4}-4 g_{A}^{2}-1\right)+\mathbf{q}^{2}\left(23 g_{A}^{4}-10 g_{A}^{2}-1\right)+\frac{48 g_{A}^{4} M_{\pi}^{4}}{4 M_{\pi}^{2}+\mathbf{q}^{2}}\right]\right. \\
& \left.+\mathbf{q}^{2} \ln \frac{M_{\pi}}{M_{\pi}^{\text {phys }}}\left(23 g_{A}^{4}-10 g_{A}^{2}-1\right)\right\} \\
& -\frac{3 g_{A}^{4}}{64 \pi^{2} F_{\pi}^{4}}\left(L(q)+\ln \frac{M_{\pi}}{M_{\pi}^{p h y s}}\right)\left\{\sigma_{1} \cdot \mathbf{q} \sigma_{2} \cdot \mathbf{q}-\mathbf{q}^{2} \sigma_{1} \cdot \sigma_{2}\right\},
\end{aligned}
$$




$$
\begin{aligned}
V^{\text {cont }}= & \bar{C}_{S}+\bar{C}_{T}\left(\sigma_{1} \cdot \sigma_{2}\right)+M_{\pi}^{2}\left(\bar{D}_{S}-\frac{3 g_{A}^{2}}{32 \pi^{2} F_{\pi}^{4}}\left(8 F_{\pi}^{2} C_{T}-5 g_{A}^{2}+2\right) \ln \frac{M_{\pi}}{M_{\pi}^{\text {phys }}}\right) \\
& +M_{\pi}^{2}\left(\bar{D}_{T}-\frac{3 g_{A}^{2}}{64 \pi^{2} F_{\pi}^{4}}\left(16 F_{\pi}^{2} C_{T}-5 g_{A}^{2}+2\right) \ln \frac{M_{\pi}}{M_{\pi}^{\text {phys }}}\right)\left(\sigma_{1} \cdot \sigma_{2}\right) \\
& +C_{1} \mathbf{q}^{2}+C_{2} \mathbf{k}^{2}+\left(C_{3} \mathbf{q}^{2}+C_{4} \mathbf{k}^{2}\right)\left(\sigma_{1} \cdot \sigma_{2}\right) \\
& +i C_{5} \frac{\sigma_{1}+\sigma_{2}}{2} \cdot(\mathbf{k} \times \mathbf{q})+C_{6}\left(\mathbf{q} \cdot \sigma_{1}\right)\left(\mathbf{q} \cdot \sigma_{2}\right)+C_{7}\left(\mathbf{k} \cdot \sigma_{1}\right)\left(\mathbf{k} \cdot \sigma_{2}\right),
\end{aligned}
$$

with $g_{A}$ and $F_{\pi}$ the physical values of the nucleon axial coupling and pion decay constant, respectively and $\bar{d}_{18}$ a low-energy constant related to the Goldberger-Treiman discrepancy 14]. The symbols $\sigma_{i}\left(\boldsymbol{\tau}_{i}\right), i=1,2$ indicate the spin (isospin) operators for particle $i$ and the $C_{1, \ldots, 7}, \bar{C}_{S, T}$, and $\bar{D}_{S, T}$ are low-energy constants (LEC's) to be determined from fits to nucleon-nucleon data. Further, $\mathbf{q}$ denotes the momentum transfer of the nucleon, i.e. $\mathbf{q}=\mathbf{p}^{\prime}-\mathbf{p}$, where $\mathbf{p}^{\prime}$ and $\mathbf{p}$ are final and initial nucleon momenta, while $\mathbf{k}=\left(\mathbf{p}^{\prime}+\mathbf{p}\right) / 2$. Here and in what follows we denote the value of the variable pion mass by $M_{\pi}$ in order to distinguish it from the physical value denoted by $M_{\pi}^{\text {phys }}=139.6 \mathrm{MeV}$. Furthermore,

$$
L(q) \equiv L(|\mathbf{q}|)=\frac{\sqrt{4 M_{\pi}^{2}+\mathbf{q}^{2}}}{|\mathbf{q}|} \ln \frac{\sqrt{4 M_{\pi}^{2}+\mathbf{q}^{2}}+|\mathbf{q}|}{2 M_{\pi}}
$$

and $\Delta$ represents the relative shift in the ratio $g_{A} / F_{\pi}$ compared to its physical value:

$$
\begin{aligned}
\Delta & \equiv \frac{\left(g_{A} / F_{\pi}\right)_{M_{\pi}}-\left(g_{A} / F_{\pi}\right)_{M_{\pi}^{\text {phys }}}}{\left(g_{A} / F_{\pi}\right)_{M_{\pi}^{\text {phys }}}} \\
& =\left(\frac{g_{A}^{2}}{16 \pi^{2} F_{\pi}^{2}}-\frac{4}{g_{A}} \bar{d}_{16}+\frac{1}{16 \pi^{2} F_{\pi}^{2}} \bar{l}_{4}\right)\left(\left(M_{\pi}^{\text {phys }}\right)^{2}-M_{\pi}^{2}\right)-\frac{g_{A}^{2} M_{\pi}^{2}}{4 \pi^{2} F_{\pi}^{2}} \ln \frac{M_{\pi}}{M_{\pi}^{\text {phys }}},
\end{aligned}
$$

where the low-energy constants $\bar{d}_{16}$ and $\bar{l}_{4}$ are defined as in Ref. 14]. Note that in the TPEP, we only take into account the explicit $M_{\pi}$-dependence and use the physical values for $g_{A}$ and $F_{\pi}$. This is sufficient at NLO since any shift in $g_{A}$ and $F_{\pi}$ for a different value of $M_{\pi}$ in the TPE is a $\mathrm{N}^{4} \mathrm{LO}$ effect. We also incorporate the leading isospin-breaking corrections due to the pion mass difference in the one-pion exchange potential [23] but do not consider an independent variation of $m_{u}-m_{d}$.

The constants $\bar{C}_{S, T}$ and $\bar{D}_{S, T}$ are related to the $C_{S, T}$ used in [24] via

$$
C_{S, T}=\bar{C}_{S, T}+\left(M_{\pi}^{p h y s}\right)^{2} \bar{D}_{S, T} .
$$

Note further that the short-range terms of the type $M_{\pi}^{2} \ln M_{\pi}$ in Eq. (6) result from the two-pion exchange as well as from the renormalization of the leading-order contact forces by pion loops. It is important to stress that renormalization of the LECs $C_{S}, C_{T}, C_{1, \ldots 7}$ due to pion loops does not depend on the pion mass and thus is of no relevance for this work. The potential $V\left(\mathbf{p}^{\prime}, \mathbf{p}\right)$ is multiplied by the regulating functions $f_{\mathrm{R}}(|\mathbf{p}|), f_{\mathrm{R}}\left(\left|\mathbf{p}^{\prime}\right|\right)$ in order to cut off the large momentum components in the Lippmann-Schwinger equation. In this study, we use the same exponential function $f_{R}(|\mathbf{p}|)=\exp \left[-\mathbf{p}^{4} / \Lambda^{4}\right]$ as in Ref. [25] and restrict ourselves to the cut-off $\Lambda=540 \mathrm{MeV}^{2}$.

\footnotetext{
${ }^{2}$ The cutoff dependence is mild in the very low energy regime, in which we are interested here. For a discussion of larger cutoffs, see Ref. [26].
} 
In principle, these equations determine the pion mass dependence of the chiral $N N$ potential uniquely. However, the extrapolation away from the physical pion mass generates errors. The dominating source are the constants $\bar{C}_{S, T}$ and $\bar{D}_{S, T}$ which cannot be determined independently from fits to data at the physical pion mass. A smaller effect is due to the error in the LEC $\bar{d}_{16}$ which is enhanced in Eq. (8) as one moves away from the physical pion mass. Both effects generate increasing uncertainties as one extrapolates away from the physical point. Note also that we do not include explicit $\Delta(1232)$ degrees of freedom. It would be interesting to see if and how our results would be modified in a theory with explicit $\Delta$ 's 9$]$.

Following Ref. [14], we use $\bar{d}_{16}=-1.23 \mathrm{GeV}^{-2}$ which is the average of three values given in Ref. 27] (See also Ref. 28]). The LEC $\bar{d}_{18}=-0.97 \mathrm{GeV}^{-2}$ is determined from the observed value of the Goldberger-Treiman discrepancy while the two remaining constants $\bar{D}_{S}$ and $\bar{D}_{T}$ are unknown. The size of these two constants can be constrained from naturalness arguments. In Ref. 14], it was argued that the corresponding dimensionless constants $F_{\pi}^{2} \Lambda_{\chi}^{2} \bar{D}_{S, T}$ can be expected to satisfy the bounds:

$$
-3 \leq F_{\pi}^{2} \Lambda_{\chi}^{2} \bar{D}_{S, T} \leq 3,
$$

where $\Lambda_{\chi} \simeq 1 \mathrm{GeV}$ is the chiral symmetry breaking scale. We note that Refs. [12, 13] do allow for a larger variation of these LEC's. The LEC's that we are going to choose below are within our more restrictive range. Therefore, the discussion on the appropriate scaling of the $D_{S, T}$ is not relevant for this study. Furthermore, these bounds are in agreement with resonance saturation estimates and similar conditions are obeyed by the known constants [29]. For the constants $C_{S, T}$, e.g., we find $C_{S}=-120.8 \mathrm{GeV}^{-2}$ and $C_{T}=1.8 \mathrm{GeV}^{-2}$ corresponding to the dimensionless coefficients $F_{\pi}^{2} C_{S}=-1.03$ and $F_{\pi}^{2} C_{T}=0.02$, respectively. The unnaturally small value of $F_{\pi}^{2} C_{T}$ is a consequence of the approximate Wigner $\mathrm{SU}(4)$ symmetry. (For a discussion of this issue in the pionless EFT, see Ref. [40]).

The ranges from Eq. (10) were used to estimate the extrapolation errors of two-nucleon observables like the deuteron binding energy and the spin-singlet and spin-triplet scattering lengths in Ref. [14]. In the exploratory study of the three-nucleon system [15], the mean values of these error bands were used as input for the three-body calculations in the contact EFT. Even though both scattering lengths were large for the mean values, they did not become infinite at the same value of the pion mass and there was no exact limit cycle for this choice of parameters.

Here we take a different approach and search for sets of values for $\bar{D}_{S}$ and $\bar{D}_{T}$ that lie within the bound given by Eq. (10) and cause the spin-singlet and spin-triplet scattering lengths to become infinite at the same value of the pion mass. For this purpose, it is more convenient to use the partial wave projected constants

$$
\begin{aligned}
& \bar{D}_{{ }^{S_{S_{1}}}}=4 \pi\left(\bar{D}_{S}+\bar{D}_{T}\right), \\
& \bar{D}_{{ }_{S_{\mathrm{S}}}}=4 \pi\left(\bar{D}_{S}-3 \bar{D}_{T}\right) .
\end{aligned}
$$

The dimensionless constants $\alpha_{1} \mathrm{~S}_{0}$ and $\alpha^{3 \mathrm{~S}_{1}}$ defined as

$$
\alpha^{{ }_{1} S_{0}}{ }=F_{\pi}^{2} \Lambda_{\chi}^{2} \bar{D}_{{ }_{S_{0}}} /(16 \pi) \quad \text { and } \quad \alpha^{{ }^{3} S_{1}}=F_{\pi}^{2} \Lambda_{\chi}^{2} \bar{D}_{{ }^{3} S_{1}} /(8 \pi),
$$

then satisfy the same bound as in Eq. (10), i.e.:

$$
\begin{aligned}
-3 & \leq \alpha^{3 \mathrm{~S}_{1}} \leq 3, \\
-3 & \leq \alpha^{1} \mathrm{~S}_{0} \leq 3 .
\end{aligned}
$$




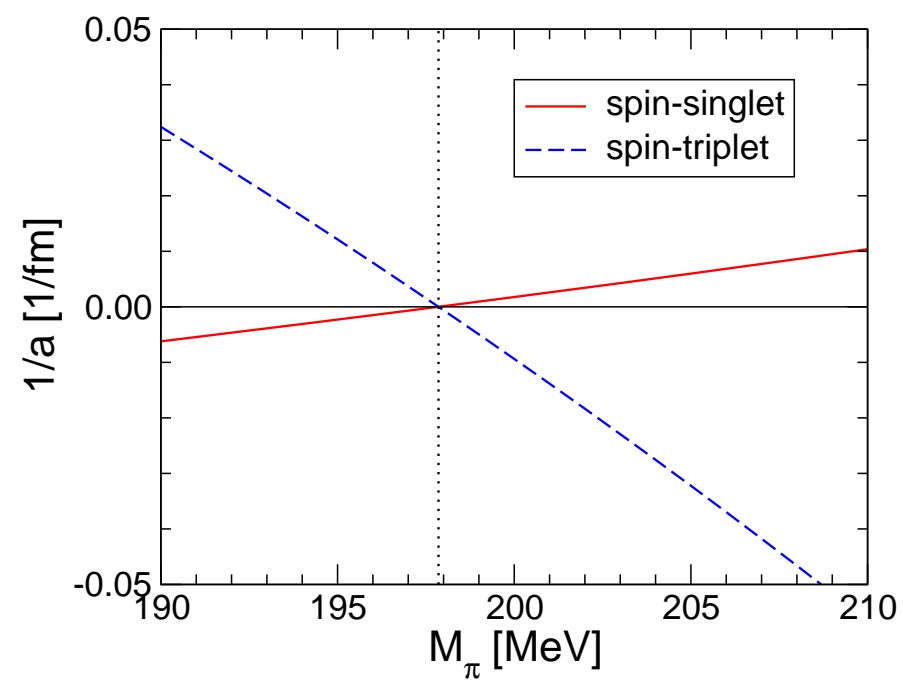

FIG. 1: Inverse of the S-wave scattering lengths in the spin-triplet and spin-singlet nucleon-nucleon channels for solution (a) as a function of the pion mass $M_{\pi}$. The vertical dotted line indicates the critical pion mass $M_{\pi}^{\text {crit }}$.

The values of the parameters $\alpha_{3} S_{1}$ and $\alpha_{1} S_{0}$ can be chosen independently anywhere in the above intervals without violating naturalness and without affecting physics at the physical value of the pion mass.

The values of the pion mass where the inverse scattering lengths in the spin-triplet and spin-singlet channels vanish simultaneously are called critical pion masses $M_{\pi}^{\text {crit }}$. At NLO in the chiral counting, it is possible to find parameter sets with critical pion masses in the range $175 \mathrm{MeV} \lesssim M_{\pi}^{\text {crit }} \lesssim 205 \mathrm{MeV}$. For example, for the three exemplifying values $\alpha_{3 \mathrm{~S}_{1}}= \pm 2.5$ and 0.0 , we obtain the following critical parameter sets:

(a) $\alpha^{3_{\mathrm{S}_{1}}}=-2.5$ and $\alpha_{1} \mathrm{~S}_{0}=2.138598 \quad \Longrightarrow \quad M_{\pi}^{\text {crit }}=197.8577 \mathrm{MeV}$,

(b) $\alpha_{{ }^{3} \mathrm{~S}_{1}}=0.0$ and $\alpha_{{ }_{1} \mathrm{~S}_{0}}=1.955709 \quad \Longrightarrow \quad M_{\pi}^{\text {crit }}=186.3276 \mathrm{MeV}$,

(c) $\alpha_{{ }_{3} S_{1}}=2.5$ and $\alpha_{1} S_{S_{0}}=1.776665 \quad \Longrightarrow \quad M_{\pi}^{\text {crit }}=179.0417 \mathrm{MeV}$.

We note that it is unlikely that physical QCD will correspond to any of the solutions (a)-(c). However, in Ref. [15] it was conjectured that one should be able to reach the critical point by varying the up- and down-quark masses $m_{u}$ and $m_{d}$ independently because the spin-triplet and spin-singlet channels have different isospin. A more detailed investigation is needed in order to test this conjecture. However, many aspects of the limit cycle are universal and do not depend on the exact parameter values [22]. Therefore, we study the structure of the three-nucleon system near the critical pion mass for solution (a) in the remainder of this paper in more detail. The universal aspects of the three-body observables do not depend on the details of the solution we choose.

The inverse scattering lengths in the spin-triplet and spin-singlet channels in the vicinity of the limit cycle for solution (a) are shown in Fig. 1. As promised, the inverse scattering lengths vanish at the critical value of the pion mass $M_{\pi}^{\text {crit }}=197.8577 \mathrm{MeV}$. For pion masses below the critical value, the spin-triplet scattering length is positive and the deuteron is bound. As the inverse spin-triplet scattering length decreases, the deuteron becomes more 


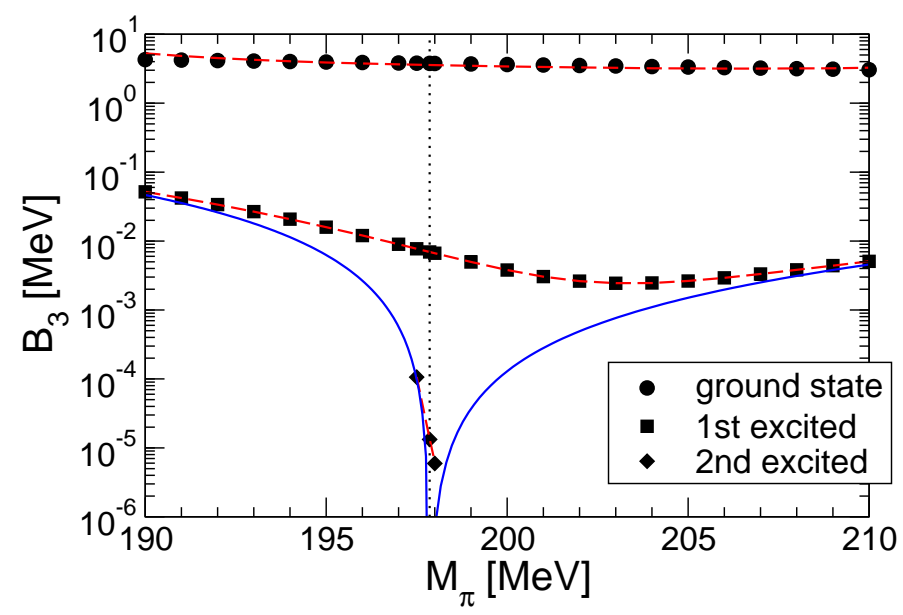

FIG. 2: Binding energies $B_{3}$ of the triton ground state and the first two excited states as function of the pion mass $M_{\pi}$. The circles (ground state), squares (first excited state), and diamonds (second excited state) give the chiral EFT result, while the dashed lines are calculations in the pionless theory. The vertical dotted line indicates the critical pion mass $M_{\pi}^{\text {crit }}$. The thresholds for the three-body states are given by the solid lines.

and more shallow and finally becomes unbound at the critical mass. Above the critical pion mass the deuteron exists as a shallow virtual state. In the spin-singlet channel, the situation is reversed: the "spin-singlet deuteron" is a virtual state below the critical pion mass and becomes bound above. The pion mass dependence of the two scattering lengths shown in Fig. 1] will be used as input for the calculations in the contact EFT in the next section.

From the solution of the Faddeev equations with solution (a) for the $N N$ potential, we obtain the binding energies of the triton and the first two excited states in the vicinity of the limit cycle (See Ref. [30] for details). The binding energies are given in Fig. 2] by the circles (ground state), squares (first excited state), and diamonds (second excited state). The solid lines indicate the neutron-deuteron $\left(M_{\pi} \leq M_{\pi}^{\text {crit }}\right)$ and neutron-spin-singlet-deuteron $\left(M_{\pi} \geq M_{\pi}^{\text {crit }}\right)$ thresholds where the three-body states become unstable. Directly at the critical mass, these thresholds coincide with the three-body threshold and the triton has infinitely many excited states. The dashed lines are calculations in the pionless theory and will be discussed in detail below. The binding energy of the triton ground state varies only weakly over the whole range of pion masses and is about one half of the physical value at the critical point. The excited states are influenced by the thresholds and vary much more strongly.

In the remainder of this subsection, we calculate the expectation values of the $2 N$ and $3 N$ kinetic energies and some properties of the $2 N$ and $3 N$ wave functions. While these quantities are technically not observables, they shed some light on the structure of the three-body states.

In Fig. 3. we show the expectation values of the kinetic energy for the triton ground and first excited states and for the two-nucleon states as a function of the pion mass $M_{\pi}$. All expectation values are evaluated in the rest frame of the corresponding states. The triton ground state kinetic energy stays fairly constant as $M_{\pi}$ is varied. The kinetic energy of the first excited state, however, approaches the kinetic energy of the two-nucleon bound state (the deuteron for $M_{\pi}<M_{\pi}^{\text {crit }}$ and the spin-singlet deuteron for $M_{\pi}>M_{\pi}^{\text {crit }}$ ) near the value 


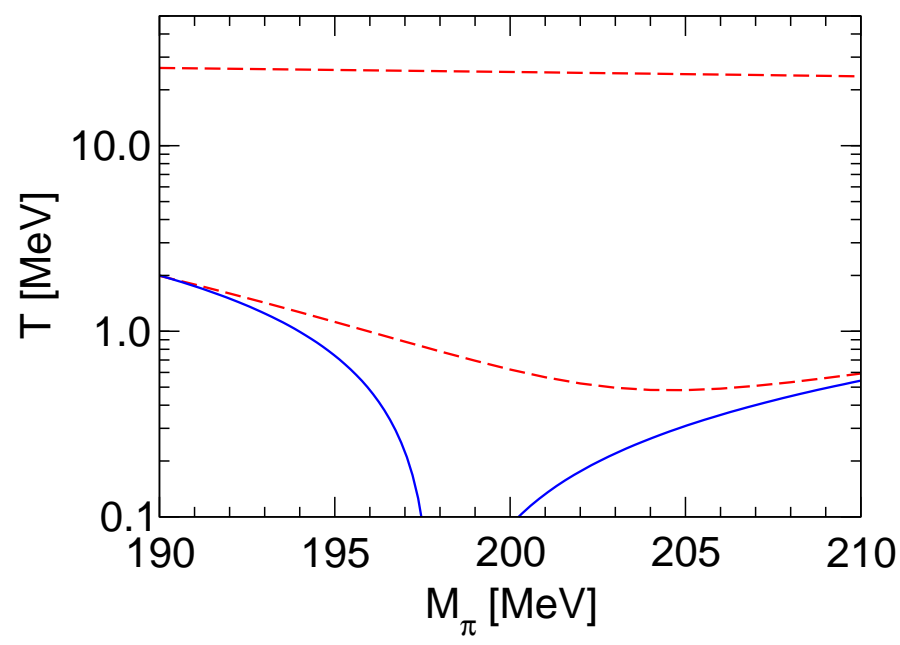

FIG. 3: Kinetic energies of the triton ground state and the first excited state indicated by the dashed lines compared to the kinetic energies of the deuteron $\left(M_{\pi}<M_{\pi}^{c r i t}\right)$ and the spin-singlet deuteron $\left(M_{\pi}>M_{\pi}^{c r i t}\right)$ indicated by the solid lines as function of pion mass $M_{\pi}$.

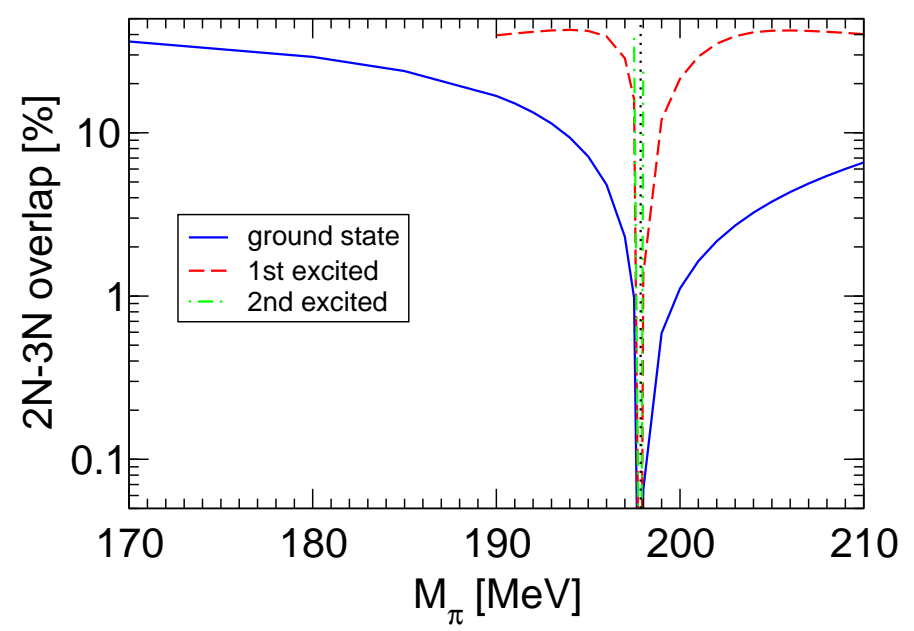

FIG. 4: $2 N-3 N$ wave function overlap for the triton ground state and the first two excited states as function of pion mass $M_{\pi}$. The vertical dotted line indicates the critical pion mass $M_{\pi}^{\text {crit }}$.

of the pion mass where the triton excited state enters from the $2 N-N$ continuum. As a consequence, the third particle is essentially at rest in this region. This behavior indicates that the first excited state has a $2 N-N$ cluster structure close to the $2 N-N$ threshold. A similar observation applies to the second excited state which is not shown in the figure.

The $2 N-3 N$ wave function overlap for the triton ground state and the first two excited states is shown in Fig. 4 as function of pion mass $M_{\pi}$. The overlap rapidly approaches zero as the pion mass reaches the critical value. This is a consequence of increasing the size of the deuteron $\left(M_{\pi} \leq M_{\pi}^{\text {crit }}\right)$ and spin-singlet-deuteron $\left(M_{\pi} \geq M_{\pi}^{\text {crit }}\right)$ as the critical point is approached from below and above, respectively. Directly at the critical point, they are both infinitely large. While new shallow three-body states appear at values of $B_{3}$ that form a geometric series and differ by factors of $\lambda_{0}^{2}=515$ as $M_{\pi}^{\text {crit }}$ is approached, the size of a given three-body state remains finite (cf. Fig. 2). Consequently, the overlap between $2 N$ and $3 N$ 
wave functions vanishes at the critical point.

We have also calculated the probability for the D-wave and mixed symmetry states in the triton ground state and the first two excited states as function of pion mass $M_{\pi}$. These results will be discussed below in the context of the contact EFT.

\section{CONTACT EFFECTIVE FIELD THEORY}

In principle, one could calculate all three-nucleon observables in the vicinity of the critical pion mass in the chiral EFT. However, the computational effort is significant, even in the $3 \mathrm{~N}$ system. Moreover, the calculations become increasingly difficult for the shallow excited states near the limit cycle. The physics near the limit cycle, however, can also be described in the contact EFT for large scattering length. This theory is formulated in an expansion around the limit of two-body bound states at threshold corresponding to vanishing inverse scattering lengths. It is much simpler than the chiral EFT and does not contain pion degrees of freedom. Three-body calculations can typically be carried out on a personal computer. Therefore, it is an ideal tool to calculate physical observables in the critical region where the scattering lengths are large. We note that the contact EFT is universal and does not have the explicit pion mass dependence. When the pion mass dependence of the spin-triplet and spin-singlet scattering lengths (cf. Fig. 1) as well as one three-body observable (cf. Fig. 2) are taken from the chiral EFT calculation, the pion mass dependence of other three-body observables can be calculated. Therefore, it complements the chiral EFT study from the previous section.

For practical purposes, it is convenient to write down this theory in the Lagrangian formalism using so-called "dibaryon" fields. In our case, we need two dibaryon fields: (i) a field $t_{i}$ with spin (isospin) 1 (0) representing two nucleons interacting in the ${ }^{3} S_{1}$ channel (the deuteron) and (ii) a field $s_{a}$ with spin (isospin) 0 (1) representing two nucleons interacting in the ${ }^{1} S_{0}$ channel [17]:

$$
\begin{array}{r}
\mathcal{L}_{\text {contact }}= \\
N^{\dagger}\left(i \partial_{t}+\frac{\nabla^{2}}{2 M}\right) N+\frac{g_{t}}{2} t_{i}^{\dagger} t_{i}+\frac{g_{s}}{2} s_{a}^{\dagger} s_{a}-\frac{g_{t}}{2}\left(t_{i}^{\dagger} N^{T} \tau_{2} \sigma_{i} \sigma_{2} N+\text { h.c. }\right) \\
-\frac{g_{s}}{2}\left(s_{a}^{\dagger} N^{T} \sigma_{2} \tau_{a} \tau_{2} N+\text { h.c. }\right)-\frac{2 M H}{\Lambda^{2}} N^{\dagger}\left[g_{t}^{2}\left(t_{i} \sigma_{i}\right)^{\dagger}\left(t_{j} \sigma_{j}\right)\right. \\
\left.+\frac{g_{t} g_{s}}{3}\left(\left(t_{i} \sigma_{i}\right)^{\dagger}\left(s_{a} \tau_{a}\right)+\text { h.c. }\right)+g_{s}^{2}\left(s_{a} \tau_{a}\right)^{\dagger}\left(s_{b} \tau_{b}\right)\right] N,
\end{array}
$$

where $i, j$ are spin and $a, b$ are isospin indices, $M$ is the nucleon mass, and $g_{t}, g_{s}$, and $H$ are the bare coupling constants. The $\sigma_{i}\left(\tau_{a}\right)$ are Pauli matrices acting in spin (isospin) space. In the two-body sector, the exact solution of the field theory can be obtained analytically [31]. Renormalization can be implemented by adjusting the two-body coupling constants $g_{t}(\Lambda)$ and $g_{s}(\Lambda)$ as a function of the ultraviolet momentum cutoff $\Lambda$ such that the spin-triplet and spin-singlet scattering lengths have the desired values. Other two-body observables are then independent of $\Lambda$ and have the appropriate values up to corrections of order $r_{0} /|a|$ and $r_{0} \sqrt{M|E|}$ where $r_{0}$ is the range of the interaction and $E$ the typical energy.

In the three-body sector, the nonperturbative solution of the field theory can be obtained by solving generalized Skorniakov-Ter-Martirosian integral equations including a three-body force numerically. These integral equations have unique solutions only in the presence of an ultraviolet cutoff $\Lambda$. The resulting predictions for three-body observables, although finite, depend on the cutoff and are periodic functions of $\ln (\Lambda)$ with period $\pi / s_{0}$ where $s_{0}=$ 


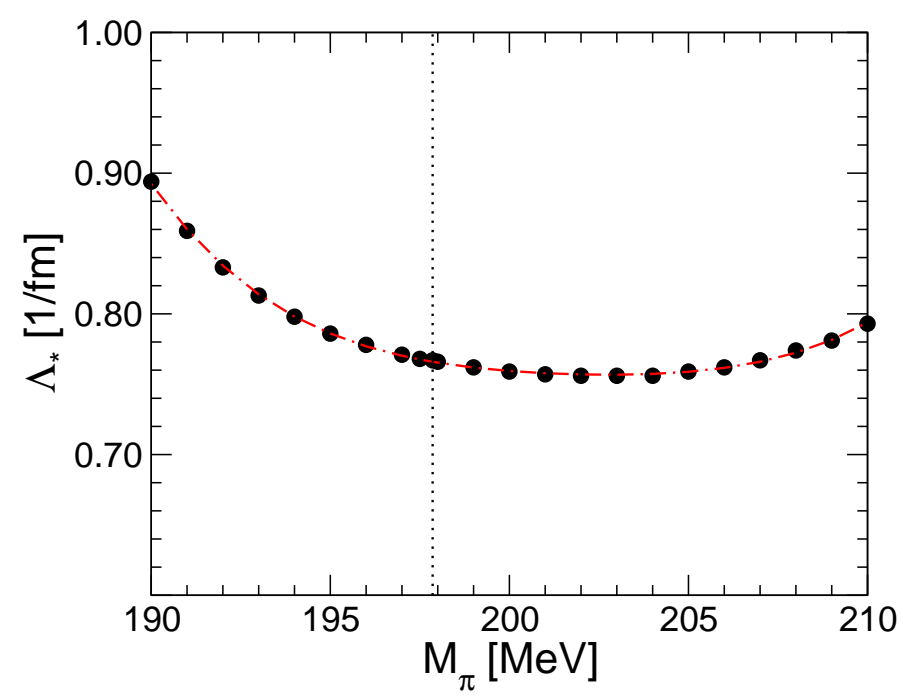

FIG. 5: The three-body parameter $\Lambda_{*}$ determined from matching the first excited state of the triton as a function of the pion mass $M_{\pi}$ (circles). The dash-dotted line is a fourth order polynomial fit while the vertical dotted line indicates the critical pion mass $M_{\pi}^{\text {crit }}$.

$1.0062378 \ldots$ is a transcendental number. In Ref. 17] it was shown that the quantum field theory could be fully renormalized to remove the residual dependence on $\Lambda$ in the threebody sector by adding a three-body interaction term to the Lagrangian density in (14). The dependence of three-body observables on the cutoff decreases like $1 / \Lambda^{2}$ if the three-body coupling constant has the form 32 ]

$$
H(\Lambda)=\frac{\cos \left[s_{0} \ln \left(\Lambda / \Lambda_{*}\right)+\arctan \left(s_{0}\right)\right]}{\cos \left[s_{0} \ln \left(\Lambda / \Lambda_{*}\right)-\arctan \left(s_{0}\right)\right]},
$$

for some value of $\Lambda_{*}$. With this renormalization, three-body observables have well-defined limits as $\Lambda \rightarrow \infty$, but they depend on the parameter $\Lambda_{*}$ introduced by dimensional transmutation. Since $H(\Lambda)$ is a periodic function of $\ln (\Lambda)$, the renormalization of the field theory involves an ultraviolet limit cycle. This EFT has succesfully been applied to various nuclear three-body observables [9]. Higher order corrections can be calculated as well [33, 34, 35, 36] but are suppressed by $r_{0} /|a|$ and $r_{0} \sqrt{M|E|}$ near the exact limit cycle. (For a recent formal study of the corrections in repulsive partial waves, see Ref. [37].)

As discussed above, we take the pion mass dependence of the spin-singlet and spin-triplet scattering lengths from the chiral EFT calculation (cf. Fig. 11). The pion mass dependence of the three-body parameter $\Lambda_{*}$ can be determined from matching the energy of the triton ground state or one of its excited states (cf. Fig. 2). Since the higher-order effects are generally smaller for the shallower states, we match to the first excited state. The pion mass dependence of all other three-body observables can then be predicted to leading order in the power counting. In Fig. 5, the circles give the value of $\Lambda_{*}$ obtained from the matching procedure as a function of the pion mass. The pion mass dependence of $\Lambda_{*}$ is well described by a fourth order polynomial fit indicated by the dash-dotted line. For pion masses between 190 and $210 \mathrm{MeV}$, the parameter $\Lambda_{*}$ varies smoothly by about $15 \%$. The $15 \%$ variation in $\Lambda_{*}$ corresponds to a variation of the triton ground state energy by about $25 \%$ over the same range of pion masses. In the previous work of Ref. [15], the value of $\Lambda_{*}$ was approximated by a constant since no three-body calculations with chiral potentials were available. 


\begin{tabular}{|c|c|c|c|c|}
\hline \hline$n$ & $B_{3}^{(n)}[\mathrm{MeV}]$ (chir.) & $B_{3}^{(n)}[\mathrm{MeV}]$ (cont.) & $B_{3}^{(n-1)} / B_{3}^{(n)}($ chir. $)$ & $B_{3}^{(n-1)} / B_{3}^{(n)}$ (cont.) \\
\hline-1 & & $1.8437 \times 10^{3}$ & & 515.0 \\
0 & 3.7736 & 3.5798 & & 515.0 \\
1 & $6.9504 \times 10^{-3}$ & $6.9504 \times 10^{-3}$ & 542.9 & 515.0 \\
2 & $1.3287 \times 10^{-5}$ & $1.3495 \times 10^{-5}$ & 523.1 & 515.0 \\
3 & - & $2.6202 \times 10^{-8}$ & - & 515.0 \\
4 & - & $5.0874 \times 10^{-11}$ & - & 515.0 \\
5 & - & $9.8779 \times 10^{-14}$ & - & 515.0 \\
6 & - & $1.9179 \times 10^{-16}$ & - & 515.0 \\
\hline \hline
\end{tabular}

TABLE I: Binding energies $B_{3}^{(n)}$ of the triton excited states and their ratios at the critical pion mass. The second and 4th columns show the results from chiral EFT while the third and 5th columns show the results from contact EFT. The dashes indicate entries that have not been calculated, while the blank entries are not defined.

We are now in the position to calculate the pion mass dependence of the other threenucleon observables. The results for triton ground state and second excited state are compared to the chiral EFT results in Fig 2, The circles (ground state), squares (first excited state), and diamonds (second excited state) give the chiral EFT result, while the dashed lines are calculations in the contact EFT. We find good agreement between the chiral and contact EFT calculations which is generally better for the shallower states. The typical error is about $10 \%$ for the ground state and below $1 \%$ for the second excited state. The first excited state is reproduced exactly because of the matching procedure.

The pionless theory can also be used to calculate the binding energies of the next few excited states in the critical region where it is very difficult to perform calculations in the chiral EFT. Our results for the binding energies of the first 6 excited states at the critical pion mass are compared to the results from chiral EFT in Table I. The second and 4th columns show the binding energies and ratios from chiral EFT while the third and 5th columns show the binding energies and ratios from contact EFT, respectively. The dashes indicate entries that have not been calculated, while the blank entries are not defined. From the ratios of the chiral EFT results it is evident that the first two excited states are still influenced by higher-order effects such as the finite range of the chiral potential. The exact ratio of $515.035 \ldots$ will be reached for the shallower states, but their calculation in the chiral EFT is computationally very expensive. The contact EFT, on the other hand, has an limit cycle in the ultraviolet by construction. If the theory is tuned to the critical point, the limit cycle is exact for all energies. The ratio in the 5th column of Table $\llbracket$ is therefore 515.035... for all states. The contact theory is most accurate for the shallower states where the chiral EFT also approaches a limit cyle and becomes less accurate for the deeper states. Note also that this theory predicts infinitely many deeper states whose binding energies are beyond the range of validity of this EFT. As an example, we have shown the state with $n=-1$ in Table 【]

The binding energies of the excited states directly at the critical point can also be obtained 


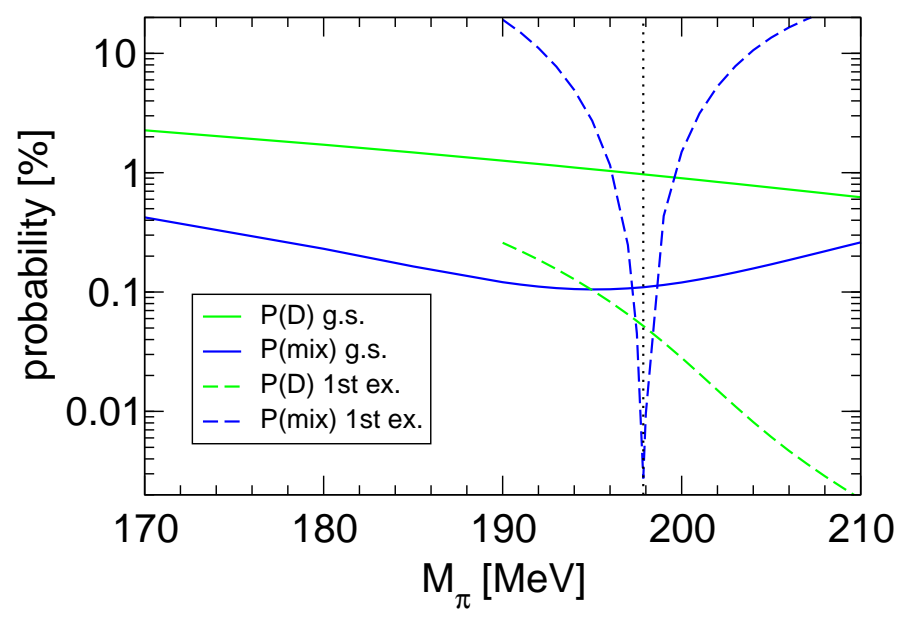

FIG. 6: Probability of the D-wave and mixed symmetry states for the triton ground state and the first excited state as function of pion mass $M_{\pi}$. The vertical dotted line indicates the critical pion $\operatorname{mass} M_{\pi}^{\text {crit }}$.

analytically from the formula [18]

$$
B_{3}^{(n)}=\left(e^{2 \pi / s_{0}}\right)^{1-n} B_{3}^{(1)}=(515.035 . .)^{1-n} B_{3}^{(1)},
$$

and the numerical results of the contact EFT are in good agreement with this analytical formula.

If one is interested in small deviations from the exact limit cycle, for example to calculate observables in the critical region, precise numerical techniques are required. Results with 5 digits of precision as in this work can be obtained in a straighforward way. Higher numerical accuracy requires more advanced techniques as described in Ref. [38], where three-body binding energies up to 12 digits of precision have been obtained for the bosonic problem. Moreover, the leading dependence of the three-body energies on the physical ultraviolet cutoff, provided by the long-range one-pion exchange in our case, was also given in Ref. [38]: A renormalization group analysis suggests that the leading corrections to the three-body binding energies $B_{3}^{(n)}$ are of order $\sqrt{B_{2}} \ln (\Lambda) / \Lambda$ and $B_{3}^{(n)} \ln (\Lambda) / \Lambda^{2}$ relative to $B_{3}^{(n)}$ itself. The first correction proportional to $\sqrt{B_{2}}$ vanishes at the critical point. The second correction

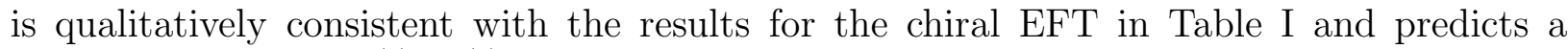
decreased value for $B_{3}^{(2)} / B_{3}^{(3)}$. However, the decrease is too strong and would lead to a ratio $B_{3}^{(2)} / B_{3}^{(3)}<515$, so other corrections must be important.

It is interesting to compare the probability for the D-wave and mixed symmetry states in the chiral EFT with the expectations from the contact EFT. In Fig. 6, we show the probability for the D-wave and mixed symmetry states in the triton ground state and the first excited state as function of pion mass $M_{\pi}$ for the chiral EFT. The probabilities for the second excited state have also been calculated but are not shown. They behave similar to the first excited state. The mixed symmetry probabilities in the triton excited states become very small near the critical pion mass. This is a consequence of the $S U(4)$ symmetry at the critical point, which leads to a decoupling of the mixed symmetry state [17].

In the chiral EFT, the $S U(4)$ symmetry at the critical point is exactly realized for the scattering lengths, but not for the effective ranges. This suggests that the mixed symmetry component is induced in the contact EFT by higher order terms proportional to $r_{e} \sqrt{M E}$. 


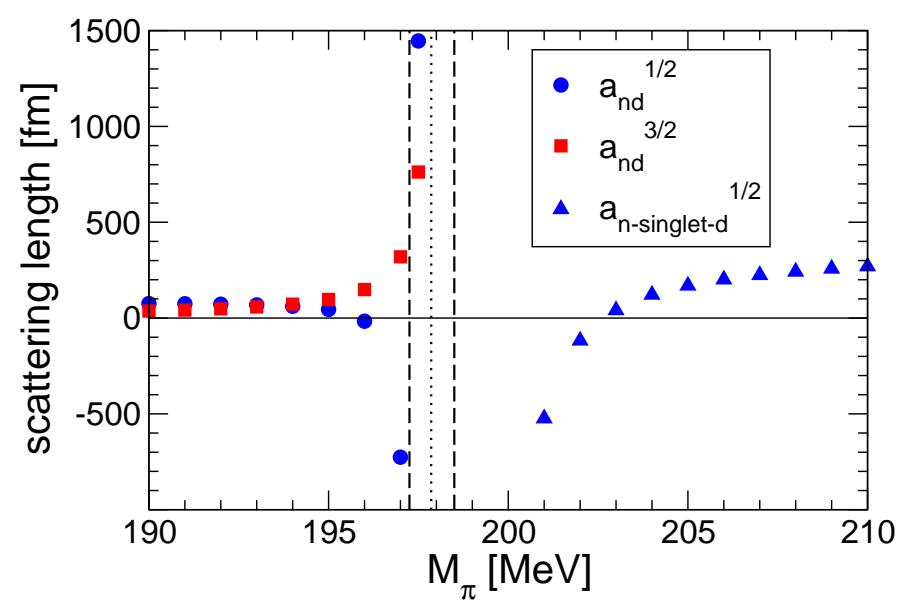

FIG. 7: The $S=1 / 2$ and $S=3 / 2$ neutron-deuteron scattering lengths $\left(M_{\pi} \leq M_{\pi}^{\text {crit }}\right)$ and the $S=1 / 2$ neutron-singlet-deuteron scattering length $\left(M_{\pi} \geq M_{\pi}^{\text {crit }}\right)$ as function of pion mass $M_{\pi}$. The vertical dotted line indicates the critical pion mass $M_{\pi}^{\text {crit }}$ while the vertical dashed lines show where the second excited state appears in the spectrum.

The observed suppression of the mixed symmetry component at the critical point is indeed consistent with this result of the power counting. The probabilities decrease roughly by factors of 30, which is in the order of magnitude expected from the binding energy ratios of the states. The strong enhancement of the mixed symmetry state probabilities once the pion mass is tuned away from the critical point is generated by $\mathrm{SU}(4)$ breaking of the scattering lengths, which is a leading-order effect in the contact EFT. We observe that the mixed symmetry state is very large, for the excited states. Treating SU(4) breaking interactions as a perturbation, this behavior might be explained by the proximity of other (virtual) excited states, which leads to an enhancement of the perturbative contributions to the wave function.

For the D-state probabilities, similar observations hold. However, since the D-state component is generated by subleading interactions for all pion masses, we do not observe the strong enhancement, once the pion mass is tuned away from the critical mass. All D-state probabilities decrease slowly with increasing pion mass. This indicates that the tensor force becomes less important at larger pion masses. This can be expected, since in the limit of very large pion masses, the pion becomes a heavy degree of freedom, so that the approximation by contact interactions becomes more and more accurate.

The calculation of three-body scattering observables in the contact EFT is also straightforward [9]. In Fig. [7, we show results for the $S=1 / 2$ and $S=3 / 2$ neutron-deuteron scattering lengths for $M_{\pi} \leq M_{\pi}^{\text {crit }}$. Above the critical pion mass the deuteron ceases to exist, but the spin-singlet-deuteron becomes bound. For $M_{\pi} \geq M_{\pi}^{\text {crit }}$, we also show the $S=1 / 2$ neutron-singlet-deuteron scattering length. The vertical dashed lines show where the second excited state appears in the spectrum. The critical point is indicated by the vertical dotted line.

All scattering lengths diverge at the critical point. The $S=3 / 2$ scattering length diverges because it is simply a constant times the spin-triplet scattering length: $1.179 a^{3} \mathrm{~S}_{1}$ [16]. The $S=1 / 2$ scattering lengths are very sensitive to the appearance of new excited states at threshold as the inverse two-body scattering lengths approach zero. They jump from $-\infty$ to $+\infty$ whenever a new state appears. 
The possible existence of a limit cycle in the pion mass region around $200 \mathrm{MeV}$ must also be taken into account in future nuclear lattice calculations. If the pion mass is in the critical region, three-body scattering observables will show a strong pion mass dependence. Since, however, the physics of the limit cycle can be captured using EFT methods as demonstrated in this work, it should not render such calculations impossible. One can simply rely on the EFT to extrapolate through the critical region to physical pion masses. A similar strategy was followed in a recent fully dynamical lattice calculation of the nucleon-nucleon scattering lengths [39].

\section{SUMMARY \& CONCLUSION}

In this paper, we have performed the first study of the conjectured infrared limit cycle in QCD in the framework of chiral EFT. We have exemplified three parameter sets in the chiral EFT at NLO that lead to an infrared limit cycle for pion masses around $200 \mathrm{MeV}$ and investigated the structure of three-body observables for one of these sets in detail.

Using both chiral and contact EFT's, we have calculated the energies and structure of the triton ground state and the first two excited states around the critical pion mass. Furthermore, we have calculated the next four excited states and the neutron-deuteron and neutron-singlet-deuteron scattering lengths in the critical region. All three-body scattering lengths diverge at the critical point. The $S=1 / 2$ scattering lengths are very sensitive to the appearance of new excited states in the critical region.

Moreover, we have elucidated the consequences for future three-body lattice QCD calculations. On the one hand, scattering observables are very sensitive to new three-body states appearing at threshold in the critical region. On the other hand, the energies of the three-body states themselves and the triton ground state energy in particular change only very slowly as one passes through the critical region. This suggests that chiral EFT could be a reliable tool for extrapolations of lattice results from unphysically large pion masses to the physical value.

The comparison of the chiral and contact EFT results also showed that an accurate matching of both theories is possible around the critical point. The small deviations of the contact EFT predictions from the full chiral result were in line with the expectations from the power counting of the contact EFT. It is reassuring to confirm these expectations by an explicit calculation.

In summary, the main findings of Ref. 15] have been confirmed and extended. Future lattice studies promise interesting insights into the rich structure of QCD. In particular, it would be very interesting to see signatures of the limit cycle in lattice simulations of the three-nucleon system with pion masses around $200 \mathrm{MeV}$ [41].

\section{Acknowledgments}

This work was supported by the U.S. Department of Energy Contract No. DE-AC0584ER40150 under which the Southeastern Universities Research Association (SURA) operates the The Thomas Jefferson National Accelerator Facility, the EU Integrated Infrastructure Initiative Hadron Physics under contract number RII3-CT-2004-506078, and the Deutsche Forschungsgemeinschaft through funds provided to the SFB/TR 16 "Subnuclear 
structure of matter". The numerical calculations have partly been performed on the JUMP cluster of the NIC, Jülich.

[1] K. G. Wilson, Rev. Mod. Phys. 55, 583 (1983).

[2] K. G. Wilson, Phys. Rev. D 3, 1818 (1971).

[3] D. J. Gross and F. Wilczek, Phys. Rev. Lett. 30, 1343 (1973); H. D. Politzer, Phys. Rev. Lett. 30, 1346 (1973).

[4] S. Weinberg, Physica A 96, 327 (1979).

[5] J. Gasser and H. Leutwyler, Nucl. Phys. B 250, 465 (1985); Annals Phys. 158, 142 (1984).

[6] V. Bernard, N. Kaiser, and U.-G. Meißner, Int. J. Mod. Phys. E 4, 193 (1995) arXiv:hep-ph/9501384.

[7] S. Weinberg, Nucl. Phys. B 363, 3 (1991); Phys. Lett. B 251, 288 (1990).

[8] S. R. Beane, P. F. Bedaque, W. C. Haxton, D. R. Phillips, and M. J. Savage, arXiv:nucl-th/0008064.

[9] P. F. Bedaque and U. van Kolck, Ann. Rev. Nucl. Part. Sci. 52, 339 (2002) arXiv:nucl-th/0203055.

[10] U.-G. Meißner, Nucl. Phys. A 751, 149 (2005) arXiv:nucl-th/0409028.

[11] E. Epelbaum, to appear in Prog. Nucl. Part. Phys. (2006) arXiv:nucl-th/0509032.

[12] S. R. Beane, P. F. Bedaque, M. J. Savage, and U. van Kolck, Nucl. Phys. A 700, 377 (2002) arXiv:nucl-th/0104030.

[13] S. R. Beane and M. J. Savage, Nucl. Phys. A 717, 91 (2003) arXiv:nucl-th/0208021; Nucl. Phys. A 713, 148 (2003) arXiv:nucl-th/0206113.

[14] E. Epelbaum, U.-G. Meißner, and W. Glöckle, Nucl. Phys. A 714, 535 (2003) arXiv:nucl-th/0207089.

[15] E. Braaten and H.-W. Hammer, Phys. Rev. Lett. 91, 102002 (2003) arXiv:nucl-th/0303038.

[16] G.V. Skorniakov and K.A. Ter-Martirosian, Sov. Phys. JETP 4, 648 (1957) [J. Exptl. Theoret. Phys. (U.S.S.R.) 31, 775 (1956)].

[17] P. F. Bedaque, H.-W. Hammer, and U. van Kolck, Nucl. Phys. A 676, 357 (2000) arXiv:nucl-th/9906032.

[18] V. N. Efimov, Sov. J. Nucl. Phys. 12, 589 (1971).

[19] V. N. Efimov, Sov. J. Nucl. Phys. 29, 546 (1979).

[20] S. Albeverio, R. Hoegh-Krohn, and T. T. Wu, Phys. Lett. A 83, 105 (1981).

[21] V. Efimov, Nucl. Phys. A 362, 45 (1981).

[22] E. Braaten and H.-W. Hammer, Phys. Rep. 428, 259 (2006) arXiv:cond-mat/0410417.

[23] U. van Kolck, J. L. Friar and T. Goldman, Phys. Lett. B 371, 169 (1996) arXiv:nucl-th/9601009.

[24] E. Epelbaum, W. Glöckle, and U.-G. Meißner, Nucl. Phys. A 671, 295 (2000) arXiv:nucl-th/9910064.

[25] E. Epelbaum, A. Nogga, W. Glöckle, H. Kamada, U.-G. Meißner, and H. Witala, Eur. Phys. J. A 15, 543 (2002) arXiv:nucl-th/0201064.

[26] A. Nogga, R.G.E. Timmermans and U. van Kolck, Phys. Rev. C 72, 054006 (2005) arXiv:nucl-th/0506005.

[27] N. Fettes, Pion-nucleon physics in chiral perturbation theory, Ph.D. Thesis, Universität Bonn, Germany, 2000, JUL-3814. 
[28] N. Fettes, V. Bernard and U.-G. Meißner, Nucl. Phys. A 669, 269 (2000) arXiv:hep-ph/9907276.

[29] E. Epelbaum, U.-G. Meißner, W. Glöckle, and C. Elster, Phys. Rev. C 65, 044001 (2002) arXiv:nucl-th/0106007.

[30] E. Epelbaum, A. Nogga, W. Glöckle, H. Kamada, U.-G. Meißner, and H. Witala, Phys. Rev. C 66, 064001 (2002) arXiv:nucl-th/0208023.

[31] D. B. Kaplan, Nucl. Phys. B 494, 471 (1997) arXiv:nucl-th/9610052.

[32] P. F. Bedaque, H.-W. Hammer, and U. van Kolck, Phys. Rev. Lett. 82, 463 (1999) arXiv:nucl-th/9809025; Nucl. Phys. A 646, 444 (1999) arXiv:nucl-th/9811046.

[33] H.-W. Hammer and T. Mehen, Phys. Lett. B 516, 353 (2001) arXiv:nucl-th/0105072.

[34] P. F. Bedaque, G. Rupak, H. W. Grießhammer, and H.-W. Hammer, Nucl. Phys. A 714, 589 (2003) arXiv:nucl-th/0207034.

[35] I. R. Afnan and D. R. Phillips, Phys. Rev. C 69, 034010 (2004) arXiv:nucl-th/0312021.

[36] H. W. Grießhammer, Nucl. Phys. A 744, 192 (2004) arXiv:nucl-th/0404073.

[37] M. C. Birse, arXiv:nucl-th/0509031.

[38] R. F. Mohr, R. J. Furnstahl, R. J. Perry, K. G. Wilson, and H.-W. Hammer, Annals Phys. 321, 225 (2006) arXiv:nucl-th/0509076.

[39] S. R. Beane, P. F. Bedaque, K. Orginos, and M. J. Savage [NPLQCD Collaboration], arXiv:hep-lat/0602010.

[40] Th. Mehen, I. W. Stewart, and M. B. Wise, Phys. Rev. Lett. 83, 931 (1999) arXiv:hep-ph/9902370.

[41] K. G. Wilson, Nucl. Phys. Proc. Suppl. 140, 3 (2005) arXiv:hep-lat/0412043. 